\title{
AN EFFICIENT DEMOSAICING TECHNIQUE USING GEOMETRICAL INFORMATION
}

\author{
Aldo Maalouf ${ }^{1}$, Mohamed-Chaker Larabi ${ }^{1}$ and Sabine Süsstrunk ${ }^{2}$ \\ ${ }^{1}$ XLIM-SIC, University of Poitiers, France \\ ${ }^{2}$ IC, EPFL, Switzerland
}

\begin{abstract}
Color image sensors use color filter arrays (CFA) to capture information at each sensor pixel position and require color demosaicing to reconstruct full color images. The quality of the demosaicked image is hindered by the sensor characteristics during the acquisition process. In this work, we propose a bandelet-based demosaicing method for color images. To this end, we have used a spatial multiplexing model of color in order to obtain the luminance and the chrominance components of the acquired image. Then, a luminance filter is used to reconstruct the luminance component. Thereafter, based on the concept of maximal gradient of multivalued images, we propose an extension of the bandelet representation for the case of multivalued images. Finally, demosaicing is performed by merging the luminance and each of the chrominance component in the multivalued bandelet transform domain. The experimental evaluation of the proposed scheme shows beneficial performance over existing demosaicing approaches.
\end{abstract}

Index Terms - Demosaicing, bandelet transform, CFA

\section{INTRODUCTION}
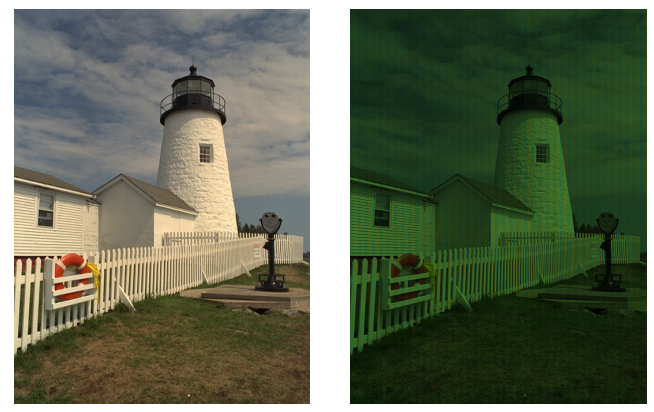

Fig. 1. Original 'lighthouse' image (left) and one color per pixel image according to the Bayer CFA (right)

As a result of the use of a color filter array (CFA), each pixel in the image has only one color component associated with it. The missing RGB values are calculated based on the neighboring pixels values by an operation called demosaicing. Demosaicing is essentially a form of interpolation which is carried out by combining the color values of pixels selected within a window around the missing value.

In general, demosaicing methods can be categorized in two groups: the first one includes the well known interpolation techniques such as nearest neighborhood, bilinear and bi-cubic interpolation while the second one regroups the methods based on inter and in-channel correlations interpolations such as the edge directed interpolation. A good overview of the demosaicing techniques can be found in [1] and [2]. Some demosaicing algorithms make use of an edge detection technique to locate the image singularities, and then perform an edge-oriented directional interpolation in order to avoid interpolation across edges [3] [1]. Other edge-directed techniques [4] estimate edges by analyzing the variance of the color differences.

Alternative methods perform separate interpolation along horizontal and vertical directions, respectively, and then, either the best reconstruction is chosen [5], [6] or a fusion of both of them is performed [7]. A number of demosaicing techniques exploit the correlation between the frequency components of the three color channels in order to estimate the missing data [8], [9]. An alternative approach consists in filtering the luminance component from the sampled color values. Then, the estimated luminance is used to reconstruct the full color image [10], [11].

This paper presents a bandelet-based scheme for the demosaicing of CFA images. The reader can refer to [12] for a full detailed description of the Bandelet transform. First, luminance and chrominance components of the CFA image are obtained by using the model proposed in [10]. Then, the luminance is interpolated and the Bandelet transform is computed. Consequently, the luminance component is segmented into a quadtree where each dyadic square regroups pixels sharing the same geometric flow direction. This geometric flow is assumed to be the same for the chromatic components. Thereafter, we propose an extension of the bandelet transform to the multispectral images. Demosaicing is accomplished by merging the luminance with each chrominance component. That is, for the three chrominance components, a multispectral image of two channels is formed (the first channel is the luminance while the second is the chrominance). The multispectral bandelet representation of the resulting image is computed. Then the inverse bandelet transform is computed to reconstruct the color image.

The remainder of the paper is organized as follows. Section 2 reviews the bandelet transform and proposes an extension to multispectral images. Section 3 is dedicated to the description of the proposed demosaicing algorithm. The experimental results are given in section 4 . Finally, this papers ends with some conclusions.

\section{MULTISPECTRAL BANDELET REPRESENTATION}

In this section, we present an extension of the bandelet transform to the case of multispectral images. The resulting multispectral representation will be used to merge the luminance component with each chrominance band in order to reconstruct the full color image. This step will be explained in the next subsection. The extension that we propose is based on the concept of the multispectral gradient defined in [13] where a first fundamental form (quadratic form) is defined for each image point. It is to be noted that the multispectral bandelet representation proposed here is not a transform, i.e. it does not allow for reconstructing the original multivalued image. It is merely a way 


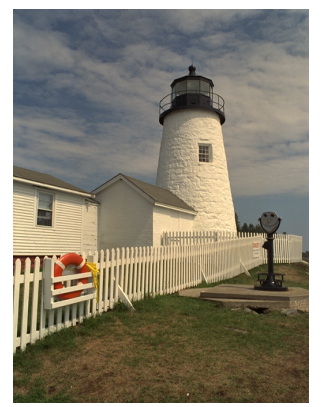

(a)

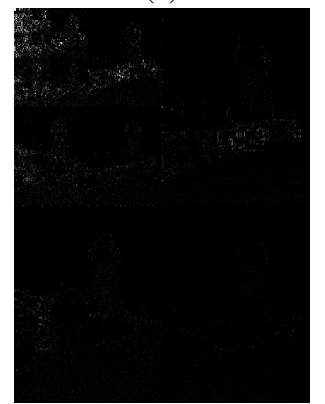

(c)

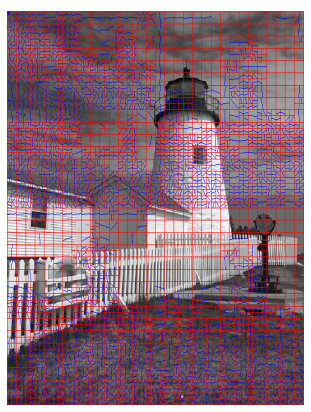

(b)

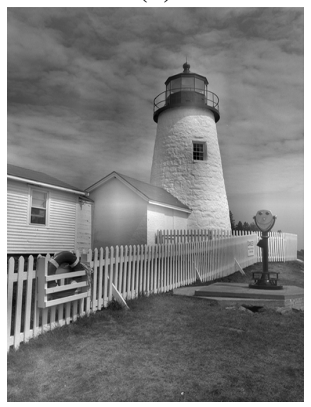

(d)
Fig. 2. (a) Lighthouse image, (b) Quadtree segmentation, (c) Multispectral bandelet representation, (d) Inverse multispectral bandelet representation

of representing combined information from different bands and this is why we refer to it by "representation". We start by an overview on the quadrature form, then we present the multispectral bandelet representation.

\subsection{Quadrature form}

In [13], Silvano di Zenzo proposed a method for computing the gradients in multivalued images. This approach can be summarized as follows.

Let $I(\mathbf{x}): \mathbb{R}^{2} \rightarrow \mathbb{R}^{m}$ be an m-band image with components for $I_{i}(\mathbf{x}): \mathbb{R}^{2} \rightarrow \mathbb{R}$ for $i=1,2,3, \ldots ., m$ ( $m=3$ for color images). Hence, at a given image location the image value is a vector in $\mathbb{R}^{m}$. Considering an infinite small displacement, the difference at two nearby points is the differential $d I$ and its squared norm is given by:

$$
\begin{aligned}
\|d I\|^{2} & =d I^{T} d I \\
& =\left(I_{x} d x+I_{y} d y\right)^{T}\left(I_{x} d x+I_{y} d y\right) \\
& =\left\|I_{x}\right\|^{2} d x^{2}+2 I_{x}^{T} I_{y} d x d y+\left\|I_{y}\right\|^{2} d y^{2} \\
& =d X^{T} G d X
\end{aligned}
$$

Where

$$
G=\sum_{i=1}^{m} \nabla I_{i} \cdot \nabla I_{i}^{T}=\left[\begin{array}{cc}
\sum_{i=1}^{m} I_{i_{x}}^{2} & \sum_{i=1}^{m} I_{i_{x}} I_{i_{y}} \\
\sum_{i=1}^{m} I_{i_{x}} I_{i_{y}} & \sum_{i=1}^{m} I_{i_{y}}^{2}
\end{array}\right]
$$

and $d X=\left(\begin{array}{c}d x \\ d y\end{array}\right)$

The extrema of the quadratic form are obtained in the direction of the eigenvectors of the matrix $G$ and the values at these loca- tions correspond with its eigenvalues $\lambda_{+}$and $\lambda_{-}$. The eigenvectors provide the direction of maximal and minimal changes at a given point in the image, and the eigenvalues are the corresponding rates of change.

Let $v_{+}$and $v_{-}$be the eigenvectors corresponding to $\lambda_{+}$and $\lambda_{-}$respectively. For a greylevel image $(m=1)$, it can be easily verified that the largest eigenvalues $\lambda_{+}=\|d I\|^{2}$ and $\lambda_{-}=0$. Therefore,

$$
\|d I(X)\|=\sqrt{\lambda_{+}} v_{+}(X)
$$

For a multispectral image, the second eigenvalue is different from zero. The first eigenvalue denotes the maximum square length of the vector $d I$ while the corresponding eigenvectors lies in the direction of the maximal length. The second eigenvector lies in the orthogonal direction. Since $\lambda_{-}$is small when compared to $\lambda_{+}$, we define

$$
d I(X) \equiv\left(\begin{array}{c}
\sqrt{\lambda_{+}} v_{+}^{1} \\
\sqrt{\lambda_{+}} v_{+}^{2}
\end{array}\right)
$$

where $v_{+}^{1}$ and $v_{+}^{2}$ are the $x$ and $y$-components of the vector $v_{+}$. Equation (4) will be used to merge the bandelet transform of the different image bands in order to obtain a single image representation. This step is explained in the next subsection.

\subsection{Multispectral Bandelet representation}

In [14], Maalouf et al. proposed a multiscale bandelet-based extension of the structure tensor (2). They showed that a better edge preserving can be obtained by substituting the horizontal and vertical derivatives by the directional derivative with respect to the direction of the geometric flow of the bandelet transform. Their multiscale multistructure tensor is defined for an $\mathrm{m}$-valued image in the bandelet domain by:

$$
G_{B}^{j}=\left[\begin{array}{cc}
\sum_{i=1}^{m}\left(\frac{\partial}{\partial x} B_{i, q}^{j} \cos \theta_{i}\right)^{2} & \sum_{i=1}^{m} \frac{\partial}{\partial x} B_{i, q}^{j} \cos \theta_{i} \frac{\partial}{\partial y} B_{i, q}^{j} \sin \theta_{i} \\
\sum_{i=1}^{m} \frac{\partial}{\partial x} B_{i, q}^{j} \cos \theta_{i} \frac{\partial}{\partial y} B_{i, q}^{j} \sin \theta_{i} & \sum_{i=1}^{m}\left(\frac{\partial}{\partial y} B_{i, q}^{j} \sin \theta_{i}\right)^{2}
\end{array}\right]
$$

The angle $\theta_{i}$ represents the angle of the optimal direction of the geometrical flow. $j$ is the scale of the $2 \mathrm{D}$ wavelet transform. $B_{i, q}^{j}$ is the corresponding bandelet coefficient at the square number $q$. The squares in the quadtree are numbered from top to bottom and from left to right.

In the following, we will use the structure tensor defined by equation (5) to merge the bandelet coefficients of the multiple image channels into one bandelet representation.

To this end, we first compute the bandelet transform of each color channel $I_{i}$. Then, the structure tensor (5) is computed and the approximation defined by (4) is used.

Since we are seeking a unique representation we have to find a "single-channel" representation $f_{g}$ having geometric structures as close as possible to the one dimensional representation of the multiband gradient (5). We can state that we wish to minimize:

$$
\min _{f_{g}} \iint\left\|G_{B}^{j}-\nabla f_{g}\right\|^{2} d x d y
$$

Equation(6) can be solved using Jacobi iteration, with homogeneous Neumann boundary conditions to ensure zero derivative at the image 
boundaries. The iteration steps are expressed as:

$$
\begin{gathered}
f_{g}^{t+1}(x, y)=\frac{1}{4}\left[f_{g}^{t}(x+1, y)+f_{g}^{t}(x-1, y)+f_{g}^{t}(x, y+1)+\right. \\
f_{g}^{t}(x, y-1)+\left\|G_{B}^{j}(x+1, y)\right\|+ \\
\left\|G_{B}^{j}(x-1, y)\right\|+\left\|G_{B}^{j}(x, y+1)\right\|+ \\
\left.\left\|G_{B}^{j}(x, y-1)\right\|\right]
\end{gathered}
$$

where $\left\|G_{B}^{j}(x, y)\right\|$ is the norm of the vector defined in (4) for the structure tensor (5) at the point $(x, y)$.

Finally, we obtain a single bandelet representation for the $m$ components of the multispectral image. Fig. 2 shows an example of the multispectral bandelet representation for a color image and the inverse bandelet transform of the obtained representation.

\section{PROPOSED DEMOSAICING TECHNIQUE}

In this section, we present the proposed demosaicing technique using the multispectral bandelet representation described in the previous section. Let $I(x, y)=[R(x, y), G(x, y), B(x, y)]$ be the original color image of the scene and $I_{C F A}(x, y)=$ $\left[R_{S}(x, y), G_{S}(x, y), B_{S}(x, y)\right]$ the CFA image. $R_{S}, G_{S}$ and $B_{S}$ are the subsampled color components. In [10], the relationship between the subsampled component and the original color components for the Bayer CFA is defined by:

$$
\begin{aligned}
& R_{S}(x, y)=\frac{1}{4} R(x, y)(1-\cos \pi x)(1+\cos \pi y) \\
& G_{S}(x, y)=\frac{1}{2} G(x, y)(1-\cos \pi x \cos \pi y) \\
& B_{S}(x, y)=\frac{1}{4} B(x, y)(1+\cos \pi x)(1-\cos \pi y)
\end{aligned}
$$

Therefore $I_{C F A}$ can be expressed as:

$$
\begin{aligned}
I_{C F A} & (x, y)=\frac{1}{4}[R(x, y)+2 G(x, y)+B(x, y)] \\
& +\frac{1}{4}[B(x, y)-R(x, y)](\cos \pi x-\cos \pi y) \\
& +\frac{1}{4}[-R(x, y)+2 G(x, y)-B(x, y)](\cos \pi x \cos \pi y)
\end{aligned}
$$

The multiplexed Bayer CFA image $I_{C F A}$ can be interpreted in the frequency domain as a luminance component at baseband and two chrominance components modulated to a higher frequency [10]. Let $L(x, y)$ the luminance component, and $I_{C_{1}}[x, y]$ and $I_{C_{2}}[x, y]$ the two chroma components we get:

$$
\begin{aligned}
I_{C F A}[x, y]= & L[x, y]+I_{C_{1}}[x, y](-1)^{x+y} \\
& +I_{C_{2}}[x, y]\left((-1)^{x}-(-1)^{y}\right)
\end{aligned}
$$

where

$$
\left(\begin{array}{c}
L \\
I_{C_{1}} \\
I_{C_{2}}
\end{array}\right)=\left(\begin{array}{ccc}
\frac{1}{4} & \frac{1}{2} & \frac{1}{4} \\
-\frac{1}{4} & \frac{1}{2} & -\frac{1}{4} \\
-\frac{1}{4} & 0 & \frac{1}{4}
\end{array}\right)\left(\begin{array}{c}
R \\
G \\
B
\end{array}\right)
$$

In order to extract the luminance $L$ we have used the filter proposed in [10]. Once we have obtained the luminance component, a bandelet decomposition is applied to obtain the quadtree segmentation $Q T_{L}$ and the bandelet transform $L_{B}$ of the Luminance. The second step consists of computing the chrominance signal by subtracting the luminance from the multiplexed signal. Then, the chrominance signal is demultiplexed by using the modulation functions proposed in [10]. Consequently, we obtain the three opponent chromatic sub-sampled signals $\left[C_{R}, C_{G}, C_{B}\right]$.

Thereafter, three 2 -valued images are formed by taking the luminance as a first channel and each of the chrominance components as a second channel. Then, for each 2-valued image, the multispectral bandelet representation presented above is computed. Here we used the quadtree $Q T_{L}$ of the luminance. We obtain three bandelet representations for each 2-valued image. Finally, the three RGB components are obtained by applying the inverse bandelet transform of the three representations.

\section{EXPERIMENTAL RESULTS}

We evaluate our demosaicing algorithm on a set of five images partly coming from state of the art database such as Lena $(512 \times 512)$, Lighthouse $(512 \times 768)$, Iris $(512 \times 512)$, Caster $(512 \times 512)$ and Haifa $(512 \times 512)$. These images have been chosen because of their content and their availability for comparisons. Fig. 3 shows an example of applying our demosaicing approach on an image.

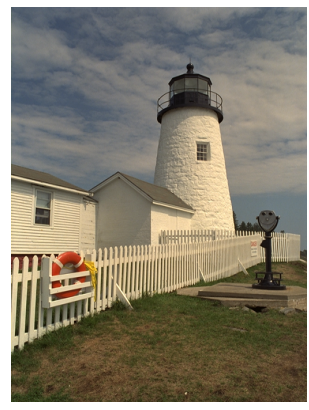

$-\mathrm{a}-$

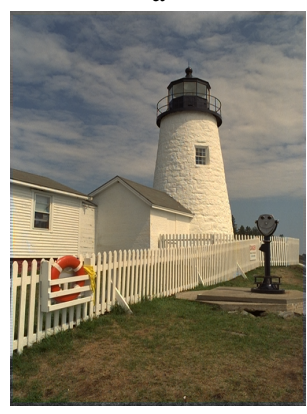

$-\mathrm{c}-$

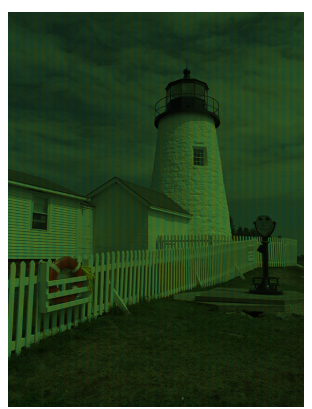

$-b-$

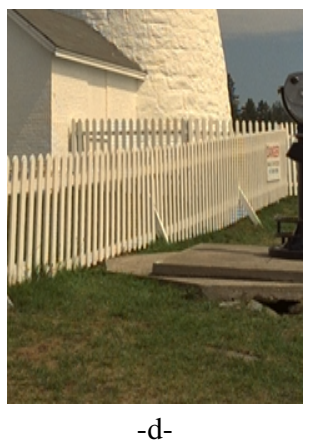

$-d-$
Fig. 3. a- Original 'lighthouse' image, b- One color per pixel image according to the Bayer CFA, c- Demosaiced image using our bandelet-based approach, d- Cropped image of the reconstructed color "lighthouse" image

First we evaluate our demosaicing algorithm and compare with the state-of-the-art techniques. Each image of the selected set is sampled according to Bayer CFA pattern. The full color representations of the color images are then reconstructed using the proposed method as well as the methods proposed in [6], [15] and [16]. The reconstructed images obtained by using the different methods are shown, for lighthouse, in figure 4. From these results, we can see that the proposed method achieved good quality reconstructed images with no zippering artifact.

In order to evaluate the quality of the reconstructed images, we have used two objective metrics: the color PSNR (CPSNR) and the Multiscale Structural Similarity Index (MS-SSIM) [17] metric. The MS-SSIM metric follows a top-down design by trying to model the physical properties of the human visual system. The MS-SSIM first decomposes images into several scales and then measures contrast and structure in each scale. In addition, the luminance of the lowest scale is also measured. Finally, all the data is pooled into a single score. MS-SSIM has the advantage that it is computationally 


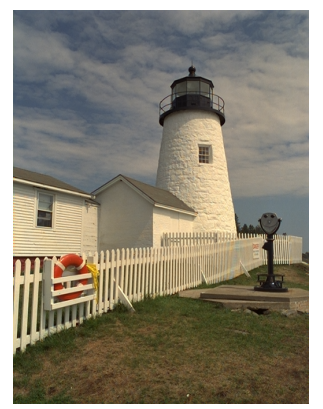

$-a-$

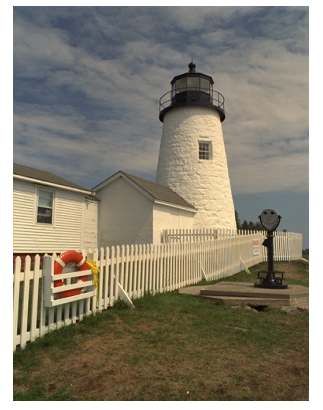

$-\mathrm{c}-$

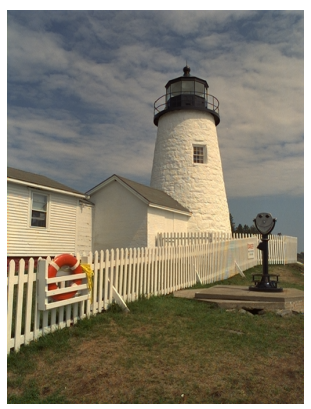

$-b-$

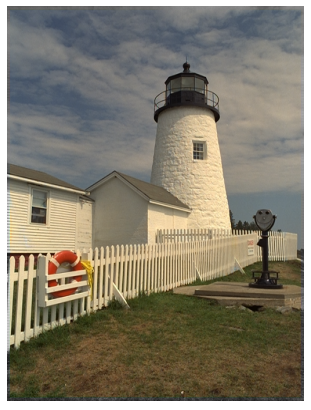

$-\mathrm{d}-$
Fig. 4. Reconstructed images using : a- method proposed in [16], bmethod proposed in [15], c- method proposed in [6], d- our method.

tractable while still providing reasonable correlations to subjective measurements [17]. The CPSNR and MS-SSIM scores are shown in table 1 from which we can see that our method achieved better scores than the state-of-the-art techniques.

\section{CONCLUSION}

In this work, we proposed a new demosaicing approach for color images. The proposed method makes use of a bandelet-based multispectral representation to represent the multivalued information of the luminance-chrominance images obtained by using the model proposed in [10]. The reconstructed image is obtained by taking the inverse bandelet transform of the proposed multispectral representation. The experimental results showed that the proposed demosaicing approach provides good results by preserving image details in comparison to literature.

Table 1. CPSNR and MS-SSIM (-20Log(1-MS-SSIM)) scores in $\mathrm{dB}$

\begin{tabular}{|c|c|c|c|c|c|}
\hline CPSNR & Lena & Lighthouse & Iris & Caster & Haifa \\
\hline Our method & $\mathbf{7 8 . 5 2}$ & $\mathbf{6 3 . 8 4}$ & $\mathbf{8 1 . 0 3}$ & $\mathbf{7 7 . 7 8}$ & $\mathbf{8 8 . 6 3}$ \\
\hline Method $[6]$ & 78.05 & 62.98 & 80.31 & 75.24 & 87.21 \\
\hline Method $[15]$ & 76.31 & 60.24 & 78.41 & 74.97 & 88.04 \\
\hline Method $[16]$ & 77.21 & 61.06 & 78.65 & 74.96 & 87.81 \\
\hline \hline MS-SSIM & Lena & Lighthouse & Iris & Caster & Haifa \\
\hline Our method & $\mathbf{8 1 . 2 3}$ & $\mathbf{7 5 . 3 6}$ & $\mathbf{8 5 . 3 4}$ & $\mathbf{8 0 . 3 1}$ & $\mathbf{9 1 . 0 6}$ \\
\hline Method [6] & 80.14 & 74.10 & 83.24 & 78.52 & 89.87 \\
\hline Method $[15]$ & 77.63 & 72.34 & 81.62 & 77.32 & 88.06 \\
\hline Method [16] & 78.35 & 73.64 & 82.06 & 77.98 & 88.50 \\
\hline
\end{tabular}

\section{REFERENCES}

[1] B. K. Gunturk, J. Glotzbach, Y. Altunbasak, R. W. Schafer, and R. M. Mersereau, "Demosaicking: Color filter array interpolation," IEEE Signal Process. Mag., vol. 22, no. 1, pp. 44-54, 2005.

[2] X. Li, B. K. Gunturk, and L. Zhang, "Image demosaicing: A systematic survey," in Proc. SPIE-IS\&T Electronic Imaging, Visual Communications and Image Processing, vol. 6822, no. 1, 2008.

[3] J. F. Hamilton and J. Adams, "Adaptive color plane interpolation in single sensor color electronic camera," U.S. patent 5 $629734,1997$.

[4] K.-H. Chung and Y.-H. Chan, "Color demosaicing using variance of color differences," IEEE Transactions on Image Processing, vol. 15, no. 10, pp. 2944-2955, 2006.

[5] K. Hirakawa and T. W. Parks, "Adaptive homogeneity-directed demosaicing algorithm," IEEE Transactions on Image Processing, vol. 14, no. 3, pp. 360-369, 2005.

[6] D. Menon, S. Andriani, and G. Calvagno, "Demosaicing with directional filtering and a posteriori decision," IEEE Transactions on Image Processing, vol. 16, no. 1, pp. 132-141, 2005.

[7] L. Zhang and X. Wu, "Color demosaicking via directional linear minimum mean square-error estimation," IEEE Trans. on Image Processing, vol. 14, no. 12, pp. 2167-2177, 2005.

[8] B. K. Gunturk, Y. Altunbasak, and R. M. Mersereau, "Color plane interpolation using alternating projections," IEEE Trans. on Image Processing, vol. 11, no. 9, pp. 997-1013, 2002.

[9] X. Li, "Demosaicing by successive approximation," IEEE Trans. on Image Processing, vol. 14, no. 3, pp. 370-379, 2005.

[10] D. Alleysson, S. Süsstrunk, and J. Hérault, "Linear demosaicing inspired by the human visual system," IEEE Transactions on Image Processing, vol. 14, no. 4, pp. 439-449, April 2005.

[11] N.-X. Lian, L. Chang, and Y.-P. Tanand V. Zagorodnov, "Adaptive filtering for color filter array demosaicking," IEEE Trans. on Image Processing, vol. 16, no. 10, pp. 2515-2525, 2007.

[12] G. Peyre and S. Mallat, "Surface compression with geometrical bandelets," ACM Trans. on Graphics, vol. 14, no. 3, 2005.

[13] DiZenzo S., "A note on the gradient of multi images," Computer Vision Graphics and Image processing, vol. 33, no. 1, pp. 116-125, 1986.

[14] Maalouf A., Carré P., Augereau B., and Fernandez-Maloigne C., "Bandelet-based anisotropic diffusion," IEEE International Conference on Image Processing (ICIP2007), San Antonio, USA, 2007.

[15] X. Li, "Demosaicing by successive approximation," IEEE Trans. on Image Processing, vol. 14, no. 3, pp. 370-379, 2005.

[16] B. K. Gunturk, Y. Altunbasak, and R. M. Mersereau, "Color plane interpolation using alternating projections," IEEE Trans. on Image Processing, vol. 11, no. 9, pp. 997-1013, 2002.

[17] Z. Wang, E.P. Simoncelli, and A.C. Bovik, "Multi-scale structural similarity for image quality assessment," IEEE Asilomar Conf. on Signals, Systems and Computers, 2003. 